\title{
Closed-form Solutions of the Cross-anisotropic Stratum Due to a Point Heat Source
}

\author{
Feng-Tsai $\operatorname{Lin}^{1}$ and John C.-C. Lu ${ }^{2}$ \\ ${ }^{1}$ Department of Naval Architecture, National Kaohsiung Marine University \\ ${ }^{2}$ Department of Civil Engineering, Chung Hua University \\ Taiwan
}

\section{Introduction}

The objective of this paper is to present the closed-form solutions of the long-term displacements and temperature change of a cross-anisotropic medium subjected to a point heat source at great depth. The medium is first assumed to be cross-anisotropic in mechanical and thermal properties. Under this assumption, the properties of the materials are different in plane of isotropy and in planes normal to it. Using Hankel and Fourier transforms, this paper presents the analytic solutions to this kind of problems, such as the repositories of nuclear wastes. The general solutions are then further simplified to cases when the material is isotropic in mechanical properties and finally totally isotropic in each property of the stratum.

Nuclear wastes are usually deposited at a great depth, such as 200 to 700 meters below ground, so that they can be isolated from the living environment of human beings. However, research efforts on the thermo-mechanical responses of soils and rocks due to the heat generated by the radioactive waste in deep underground are still very limited. Excessive thermal difference usually results in a volume change of water and solid skeleton. This change can cause an increase in excess pore water pressure, and in turn a decrease in effective stress, which can result in a thermal failure in the stratum due to the loss of shear resistance of solid skeleton.

The governing equations proposed by Biot $(1941,1955)$ for a fluid-saturated poroelastic solid in an isothermal quasi-static state also provided an excellent insight into a variety of mechanical phenomena. His theory was later re-formulated by Rice and Cleary (1976). Schiffman (1971) extended Biot's theory to take the thermal effects into account. The solutions to the thermal consolidation of a saturated elastic porous media around a point heat source were presented by Booker and Savvidou (1984, 1985), Savvidou and Booker (1989). In their solutions, the flow is considered to be isotropic (Booker and Savvidou, 1984, 1985) or cross-anisotropic (Savvidou and Booker, 1989) whereas the mechanical and thermal properties of the stratum are treated as being isotropic. However, it was found that the anisotropic property in the permeability of the soils has significant effects on the excess pore 
water pressure generated by a heat source (Savvidou and Booker, 1989). Lu and Lin (2006) displayed transient ground surface displacement produced by a point heat source/sink through analog quantities between poroelasticity and thermoelasticity. Based on Biot's three-dimensional consolidation theory of porous media, analytical solutions of the transient thermo-consolidation deformation due to a point heat source buried in a saturated isotropic porous elastic half space were presented by $\mathrm{Lu}$ and Lin (2007), Lin and $\mathrm{Lu}$ (2009). Within the framework of the linear theory of thermoelasticity, Chao, Chen and Shen (2006) discussed the problem of circularly cylindrical layered media subjected to an arbitrary point heat source.

Soils in general are deposited through a process of sedimentation over a long period of time. Under the accumulative overburden pressure, soils display significant anisotropy on mechanical, seepage and thermal properties. Both the soil and the stratified rock masses show the nature of anisotropy. For this reason, theoretical or numerical models should be able to simulate this kind of layered soils and rocks as cross-anisotropic medium (Amadei et al., 1988; Barden, 1963; Gibson, 1974; Lee \& Yang, 1998; Sekowski, 1986; Sheorey, 1994).

In this paper, the soil mass is modelled as a linearly elastic medium with cross-anisotropic properties. Both the thermal flow and the mechanical properties are assumed to be crossanisotropic. By using the Hankel and Fourier transforms, closed-form solutions of the longterm displacements and temperature change of the stratum due to a point heat source at large depth are obtained. The results are reduced to an isotropic case to provide a better understanding of the thermally induced responses of the stratum.

\section{Mathematical Model}

\subsection{Basic Equations}

Figure 1 shows a point heat source buried in a stratum at a great depth. Consider a homogeneous layer of cross-anisotropic soil or rock. For simplicity, the plane of symmetry of the stratum is in the horizontal direction. Let $(r, \theta, z)$ be a cylindrical coordinate system for this layer of solid where the plane of isotropy coincides with the horizontal (or $r-\theta$ ) plane. Let $u_{r}$ and $u_{z}$ be the displacements in the radial and vertical directions, respectively. The constitutive law for an elastic medium with linear axisymmetric deformation can thus be expressed by

$$
\begin{gathered}
\sigma_{r r}=A \frac{\partial u_{r}}{\partial r}+(A-2 N) \frac{u_{r}}{r}+F \frac{\partial u_{z}}{\partial z}-\beta_{r} \vartheta, \\
\sigma_{\theta \theta}=(A-2 N) \frac{\partial u_{r}}{\partial r}+A \frac{u_{r}}{r}+F \frac{\partial u_{z}}{\partial z}-\beta_{r} \vartheta, \\
\sigma_{z z}=F \frac{\partial u_{r}}{\partial r}+F \frac{u_{r}}{r}+C \frac{\partial u_{z}}{\partial z}-\beta_{z} \vartheta, \\
\sigma_{r z}=L\left(\frac{\partial u_{r}}{\partial z}+\frac{\partial u_{z}}{\partial r}\right),
\end{gathered}
$$

where $\sigma_{r r}, \sigma_{\theta \theta}, \sigma_{z z}$ and $\sigma_{r z}$ are the stress components, $\vartheta$ is the temperature change of the stratum, and $A, C, F, L, N$ are the material constants of a cross-anisotropic medium defined by Love (1944). In these equations, $\beta_{r}$ and $\beta_{z}$ represent the thermal expansion 
factors along and normal to the symmetric plane, respectively. For symmetric problem, it can be noted that the shear stresses $\sigma_{r \theta}, \sigma_{\theta z}$, and circumferential displacement $u_{\theta}$ would vanish as the vertical $z$-axis is located through the point heat source.

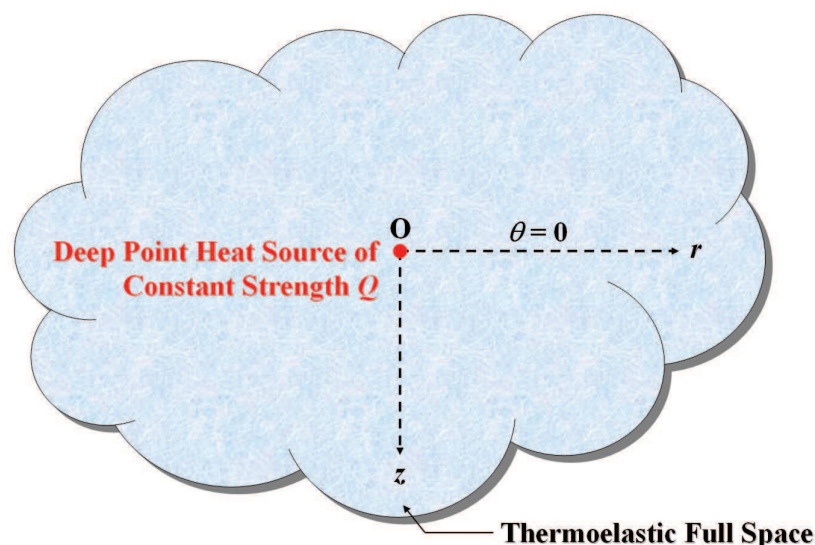

Fig. 1. Point heat source of constant heat generation rate buried deep in a cross-anisotropic stratum

Let $E_{r}$ and $E_{z}$ be Young's moduli with respect to directions lying in the plane of isotropy and perpendicular to it, respectively; $v_{r \theta}$ be Poisson's ratio for strain in the horizontal direction due to a horizontal direct stress; $v_{r z}$ be Poisson's ratio for strain in the vertical direction due to a horizontal direct stress; $v_{z r}$ be Poisson's ratio for strain in the horizontal direction due to a vertical direct stress; and $G_{r z}$ be shear modulus for planes normal to the plane of isotropy. Equations (1a)-(1d) can then be converted to

$$
\left\{\begin{array}{c}
\frac{\partial u_{r}}{\partial r} \\
\frac{u_{r}}{r} \\
\frac{\partial u_{z}}{\partial z} \\
\frac{\partial u_{r}}{\partial z}+\frac{\partial u_{z}}{\partial r}
\end{array}\right\}=\left[\begin{array}{cccc}
\frac{1}{E_{r}} & -\frac{v_{r \theta}}{E_{r}} & -\frac{v_{r z}}{E_{r}} & 0 \\
-\frac{v_{r \theta}}{E_{r}} & \frac{1}{E_{r}} & -\frac{v_{r z}}{E_{r}} & 0 \\
-\frac{v_{z r}}{E_{z}} & -\frac{v_{z r}}{E_{z}} & \frac{1}{E_{z}} & 0 \\
0 & 0 & 0 & \frac{1}{G_{r z}}
\end{array}\right]\left\{\begin{array}{c}
\sigma_{r r} \\
\sigma_{\theta \theta} \\
\sigma_{z z} \\
\sigma_{r z}
\end{array}\right\}+\left\{\begin{array}{c}
\alpha_{s r} \vartheta \\
\alpha_{s r} \vartheta \\
\alpha_{s z} \vartheta \\
0
\end{array}\right\},
$$

where $\alpha_{s r}$ and $\alpha_{s z}$ are the linear thermal expansion coefficients of the stratum in the horizontal and vertical directions, respectively. By comparison, the mechanical and thermal constants employed in equations (1a)-(1d) and (2) are related through the following equations: 


$$
\begin{gathered}
A=\frac{E_{r}\left(1-v_{r z} v_{z r}\right)}{\left(1+v_{r \theta}\right)\left(1-v_{r \theta}-2 v_{r z} v_{z r}\right)}, \\
C=\frac{E_{z}\left(1-v_{r \theta}\right)}{1-v_{r \theta}-2 v_{r z} v_{z r}}, \\
F=\frac{E_{z} v_{r z}}{1-v_{r \theta}-2 v_{r z} v_{z r}}=\frac{E_{r} v_{z r}}{1-v_{r \theta}-2 v_{r z} v_{z r}}, \\
L=G_{r z}, \\
N=\frac{E_{r}}{2\left(1+2 v_{r \theta}\right)}, \\
\beta_{r}=2(A-N) \alpha_{s r}+F \alpha_{s z}, \\
\beta_{z}=2 F \alpha_{s r}+C \alpha_{s z} .
\end{gathered}
$$

For the case of isotropy, $A=C=\lambda+2 G, F=\lambda, L=N=G$, and $\beta_{r}=\beta_{z}=(2 G+3 \lambda) \alpha_{s}$. Here, $\lambda$ and $G$ are the Lame moduli of the medium and $\alpha_{s}$ is the linear thermal expansion coefficient of the solid skeleton.

For a general problem, these stresses must satisfy the equilibrium equations $\sigma_{i j, j}+f_{i}=0$, where $f_{i}$ denotes the body forces. For axisymmetric problems and neglect the effects of body forces, the equilibrium equations can be expressed in terms of displacements $u_{i}$ and temperature change of the medium $\vartheta$ as follows:

$$
\begin{gathered}
A\left(\frac{\partial^{2} u_{r}}{\partial r^{2}}+\frac{1}{r} \frac{\partial u_{r}}{\partial r}-\frac{u_{r}}{r^{2}}\right)+L \frac{\partial^{2} u_{r}}{\partial z^{2}}+(F+L) \frac{\partial^{2} u_{z}}{\partial r \partial z}-\beta_{r} \frac{\partial \vartheta}{\partial r}=0 \\
(F+L)\left(\frac{\partial^{2} u_{r}}{\partial r \partial z}+\frac{1}{r} \frac{\partial u_{r}}{\partial z}\right)+L\left(\frac{\partial^{2} u_{z}}{\partial r^{2}}+\frac{1}{r} \frac{\partial u_{z}}{\partial r}\right)+C \frac{\partial^{2} u_{z}}{\partial z^{2}}-\beta_{z} \frac{\partial \vartheta}{\partial z}=0
\end{gathered}
$$

Using the law of conservation of energy, the equation can be obtained as listed below.

$$
-\nabla \cdot \boldsymbol{h}+q_{h}=0,
$$

where $\boldsymbol{h}$ is the heat flux vector and $q_{h}$ is the internal (or external) heat sources.

To describe the behavior of the heat flow in a cross-anisotropic medium, let $\lambda_{t r}$ denote the horizontal thermal conductivity of heat flow in the planes of isotropy and let $\lambda_{t z}$ be the corresponding vertical thermal conductivity in the plane perpendicular to the isotropic plane. Assuming that the heat flow follows Fourier's law, then

$$
\boldsymbol{h}=-\lambda_{t r} \frac{\partial \vartheta}{\partial r} \boldsymbol{i}_{r}-\lambda_{t z} \frac{\partial \vartheta}{\partial z} \boldsymbol{i}_{z}
$$


in which $\boldsymbol{i}_{r}$ and $\boldsymbol{i}_{z}$ are unit vectors parallel to the radial and vertical directions, respectively. Consider a point heat source of strength $Q$ located at point $(0,0)$ at great depth. Substituting (6) into (5) yields the third governing equation to relate $\vartheta$ as listed below:

$$
\lambda_{t r}\left(\frac{\partial^{2} \vartheta}{\partial r^{2}}+\frac{1}{r} \frac{\partial \vartheta}{\partial r}\right)+\lambda_{t z} \frac{\partial^{2} \vartheta}{\partial z^{2}}+\frac{Q}{2 \pi r} \delta(r) \delta(z)=0
$$

where $\delta(r)$ and $\delta(z)$ are the Dirac delta functions.

For a linearly elastic medium with cross-anisotropic properties, the differential equations expressed by Eqs. (4a), (4b) and (7) govern the steady state response of the medium subjected to axisymmetric and thermoelastic disturbance.

\subsection{Boundary Conditions}

Assume that the point heat source at great depth has no effect on the ground surface. This implies that the ground surface can be treated as a remote boundary and the stratum can be modeled as an infinite space. Thus the effect of the deep thermally disturbance vanishes at the remote boundaries $z \rightarrow \pm \infty$. In other words, the displacements in the radial and vertical directions, and the temperature change of the stratum at remote boundaries should be vanished. Then the remote boundary conditions can be expressed by

$$
u_{r}(r, z) \rightarrow 0, u_{z}(r, z) \rightarrow 0, \text { and } \vartheta(r, z) \rightarrow 0 \text { as } z \rightarrow \pm \infty
$$

The thermoelastic responses can be derived from the differential equations (4a), (4b) and (7) corresponding with the remote boundary conditions at $z \rightarrow \pm \infty$.

\section{Analytic Solutions}

\subsection{Hankel Transform Solutions}

The governing partial differential equations (4a), (4b) and (7) can be simplified to the ordinary differential equations by performing appropriate Hankel transforms (Sneddon, 1951) with respect to the radial coordinate $r$ of first, zeroth and zeroth orders, respectively. Therefore, we obtain

$$
\begin{gathered}
-\xi^{2} A U_{r}+L \frac{d^{2} U_{r}}{d z^{2}}-\xi(F+L) \frac{d U_{z}}{d z}+\xi \beta_{r} \Theta=0, \\
\xi(F+L) \frac{d U_{r}}{d z}-\xi^{2} L U_{z}+C \frac{d^{2} U_{z}}{d z^{2}}-\beta_{z} \frac{d \Theta}{d z}=0, \\
-\xi^{2} \lambda_{t r} \Theta+\lambda_{t z} \frac{d^{2} \Theta}{d z^{2}}=-\frac{Q}{2 \pi} \delta(z),
\end{gathered}
$$

where

$$
U_{r}(z ; \xi)=\int_{0}^{\infty} r u_{r}(r, z) J_{1}(\xi r) d r
$$




$$
\begin{aligned}
& U_{z}(z ; \xi)=\int_{0}^{\infty} r u_{z}(r, z) J_{0}(\xi r) d r \\
& \Theta(z ; \xi)=\int_{0}^{\infty} r \vartheta(r, z) J_{0}(\xi r) d r
\end{aligned}
$$

In these equations, $J_{\alpha}(x)$ represents the Bessel's function of the first kind of order $\alpha$. The displacements in the radial and vertical directions, and the temperature change of the stratum can then be obtained by inverting the equations (10a) to (10c), respectively, as shown below.

$$
\begin{aligned}
& u_{r}(r, z)=\int_{0}^{\infty} \xi U_{r}(z ; \xi) J_{1}(\xi r) d \xi, \\
& u_{z}(r, z)=\int_{0}^{\infty} \xi U_{z}(z ; \xi) J_{0}(\xi r) d \xi, \\
& \vartheta(r, z)=\int_{0}^{\infty} \xi \Theta(z ; \xi) J_{0}(\xi r) d \xi .
\end{aligned}
$$

Now further consideration to perform the Fourier transformations (Sneddon, 1951) with respect to the axial coordinate $z$ on equations (9a) to (9c). The results can be expressed as

$$
\begin{gathered}
-\left(\xi^{2} A+\omega^{2} L\right) \tilde{U}_{r}-i \omega \xi(F+L) \tilde{U}_{z}+\xi \beta_{r} \tilde{\Theta}=0, \\
i \omega \xi(F+L) \tilde{U}_{r}-\left(\xi^{2} L+\omega^{2} C\right) \tilde{U}_{z}-i \omega \beta_{z} \tilde{\Theta}=0, \\
\left(\xi^{2} \lambda_{t r}+\omega^{2} \lambda_{t z}\right) \tilde{\Theta}=\frac{Q}{2 \pi},
\end{gathered}
$$

where

$$
\left\{\tilde{U}_{r}(\xi, \omega), \tilde{U}_{z}(\xi, \omega), \tilde{\Theta}(\xi, \omega)\right\}=\int_{-\infty}^{\infty}\left\{U_{r}(z ; \xi), U_{z}(z ; \xi), \Theta(z ; \xi)\right\} e^{i \omega z} d z
$$

The closed-form solutions of the long-term thermoelastic deformations and temperature change of the cross-anisotropic medium subjected to a deep point heat source can then be easily obtained in the integral transformed domain $(\xi, \omega)$ by solving the simultaneous algebraic equations of (12a) to (12c). The results are shown as follows:

$$
\begin{gathered}
\tilde{U}_{r}(\xi, \omega)=\frac{Q}{2 \pi} \frac{\xi\left\{\xi^{2} L \beta_{r}-\left[(F+L) \beta_{z}-C \beta_{r}\right] \omega^{2}\right\}}{\left(\xi^{2} \lambda_{t r}+\omega^{2} \lambda_{t z}\right)\left\{C L \omega^{4}+[A C-F(F+2 L)] \xi^{2} \omega^{2}+A L \xi^{4}\right\}}, \\
\tilde{U}_{z}(\xi, \omega)=\frac{Q}{2 \pi} \frac{i \omega\left\{-\omega^{2} L \beta_{z}+\left[(F+L) \beta_{r}-A \beta_{z}\right] \xi^{2}\right\}}{\left(\xi^{2} \lambda_{t r}+\omega^{2} \lambda_{t z}\right)\left\{C L \omega^{4}+[A C-F(F+2 L)] \xi^{2} \omega^{2}+A L \xi^{4}\right\}} \\
\tilde{\Theta}(\xi, \omega)=\frac{Q}{2 \pi} \frac{1}{\xi^{2} \lambda_{t r}+\omega^{2} \lambda_{t z}} .
\end{gathered}
$$


These solutions can also be expressed in the domain $(z ; \xi)$ by applying the inverse of the Fourier transforms to Eqs. (14a) to (14c), or in mathematic language,

$$
\left\{U_{r}(z ; \xi), U_{z}(z ; \xi), \Theta(z ; \xi)\right\}=\frac{1}{2 \pi} \int_{-\infty}^{\infty}\left\{\tilde{U}_{r}(\xi, \omega), \tilde{U}_{z}(\xi, \omega), \tilde{\Theta}(\xi, \omega)\right\} e^{-i \omega z} d \omega
$$

With the help of any mathematical handbooks (Erdelyi et al., 1954; Gradshteyn \& Ryzhik, 1980) and some calculations, the solutions can be derived in the space domain $(r, z)$ by applying the inverse of the Hankel transforms to Eq. (15). The results are shown as following:

$$
\begin{gathered}
u_{r}(r, z)=\frac{Q}{4 \pi \lambda_{t z}}\left(a_{1} \frac{r}{R_{1}^{*}}+a_{2} \frac{r}{R_{2}^{*}}+a_{3} \frac{r}{R_{3}^{*}}\right), \\
u_{z}(r, z)=\frac{Q}{4 \pi \lambda_{t z}}\left(b_{1} \sinh ^{-1} \frac{\mu_{1} z}{r}+b_{2} \sinh ^{-1} \frac{\mu_{2} z}{r}+b_{3} \sinh ^{-1} \frac{\mu_{3} z}{r}\right), \\
\vartheta(r, z)=\frac{Q}{4 \pi \lambda_{t z}} \frac{1}{\mu_{3} R_{3}} .
\end{gathered}
$$

In these equations, $R_{i}=\sqrt{r^{2}+\mu_{i}^{2} z^{2}}$ and $R_{i}^{*}=R_{i}+\mu_{i}|z|(i=1,2,3)$. The constants $a_{i}$ and $b_{i}$ $(i=1,2,3)$ are defined by

$$
\begin{gathered}
a_{1}=\frac{L \beta_{r}+\left[(F+L) \beta_{z}-C \beta_{r}\right] \mu_{1}^{2}}{C L \mu_{1}\left(\mu_{1}^{2}-\mu_{2}^{2}\right)\left(\mu_{1}^{2}-\mu_{3}^{2}\right)}, \\
a_{2}=\frac{L \beta_{r}+\left[(F+L) \beta_{z}-C \beta_{r}\right] \mu_{2}^{2}}{C L \mu_{2}\left(\mu_{2}^{2}-\mu_{1}^{2}\right)\left(\mu_{2}^{2}-\mu_{3}^{2}\right)}, \\
a_{3}=\frac{L \beta_{r}+\left[(F+L) \beta_{z}-C \beta_{r}\right] \mu_{3}^{2}}{C L \mu_{3}\left(\mu_{3}^{2}-\mu_{1}^{2}\right)\left(\mu_{3}^{2}-\mu_{2}^{2}\right)}, \\
b_{1}=\frac{L \beta_{z} \mu_{1}^{2}+(F+L) \beta_{r}-A \beta_{z}}{C L\left(\mu_{1}^{2}-\mu_{2}^{2}\right)\left(\mu_{1}^{2}-\mu_{3}^{2}\right)}, \\
b_{2}=\frac{L \beta_{z} \mu_{2}^{2}+(F+L) \beta_{r}-A \beta_{z}}{C L\left(\mu_{2}^{2}-\mu_{1}^{2}\right)\left(\mu_{2}^{2}-\mu_{3}^{2}\right)}, \\
b_{3}=\frac{L \beta_{z} \mu_{3}^{2}+(F+L) \beta_{r}-A \beta_{z}}{C L\left(\mu_{3}^{2}-\mu_{1}^{2}\right)\left(\mu_{3}^{2}-\mu_{2}^{2}\right)} .
\end{gathered}
$$

In addition, $\mu_{1}$ and $\mu_{2}$ must satisfy the following characteristic equation:

and $\mu_{3}=\sqrt{\lambda_{t r} / \lambda_{t z}}$.

$$
C L \mu^{4}-[A C-F(F+2 L)] \mu^{2}+A L=0
$$




\subsection{Cases of Isotropic Mechanical Behavior with Cross-anisotropic Thermal \\ Properties}

The displacement and temperature change for a stratum with cross-anisotropic properties in mechanical and heat flows are analytically solved as expressed in Eqs. (16a)-(16c) under the disturbance of a deep point heat source. For the special case when the isotropic mechanical properties of the medium is introduced, the related closed-form solutions are obtained by taking the limit conditions of $\mu_{1}=\mu_{2}=1$ for Eqs. (16a)-(16c). This is carried out by using of L'Hospital's rule and careful calculations. The results are given as follows:

$$
\begin{gathered}
u_{r}(r, z)=\frac{Q}{4 \pi \eta G \lambda_{t z}}\left\{\beta_{r}^{*} \varphi_{1}(r, z)+\left[2 \eta \beta_{r}^{*}-(2 \eta-1) \beta_{z}^{*}\right] \varphi_{2}(r, z)\right\}, \\
u_{z}(r, z)=\frac{Q}{4 \pi \eta G \lambda_{t z}}\left\{\beta_{z}^{*} \varphi_{3}(r, z)+\left[2 \eta \beta_{z}^{*}-(2 \eta-1) \beta_{r}^{*}\right] \varphi_{4}(r, z)\right\}, \\
\vartheta(r, z)=\frac{Q}{4 \pi \lambda_{t z}} \frac{1}{\mu_{3} R_{3}},
\end{gathered}
$$

where $\eta=(1-v) /(1-2 v)$ and

$$
\begin{gathered}
\beta_{r}^{*}=2 G\left(\alpha_{s r}+v \alpha_{s z}\right) /(1-2 v), \\
\beta_{z}^{*}=2 G\left[2 v \alpha_{s r}+(1-v) \alpha_{s z}\right] /(1-2 v) .
\end{gathered}
$$

The functions $\varphi_{i}(i=1,2,3,4)$ in Eqs. (19a) and (19b) are functions of space variables $r$ and $z$ and defined as:

$$
\begin{gathered}
\varphi_{1}(r, z)=\frac{1}{4\left(\mu_{3}^{2}-1\right)} \frac{r}{R}-\frac{1}{2\left(\mu_{3}^{2}-1\right)^{2}} \frac{r}{R^{*}}+\frac{1}{2 \mu_{3}\left(\mu_{3}^{2}-1\right)^{2}} \frac{r}{R_{3}^{*}}, \\
\varphi_{2}(r, z)=\frac{1}{4\left(\mu_{3}^{2}-1\right)}\left(-\frac{r|z|}{R R^{*}}+\frac{r}{R^{*}}\right)+\frac{1}{2\left(\mu_{3}^{2}-1\right)^{2}} \frac{r}{R^{*}}-\frac{\mu_{3}}{2\left(\mu_{3}^{2}-1\right)^{2}} \frac{r}{R_{3}^{*}}, \\
\varphi_{3}(r, z)=-\frac{1}{4\left(\mu_{3}^{2}-1\right)} \frac{z}{R}-\frac{\mu_{3}^{2}}{2\left(\mu_{3}^{2}-1\right)^{2}} \sinh ^{-1} \frac{z}{r}+\frac{\mu_{3}^{2}}{2\left(\mu_{3}^{2}-1\right)^{2}} \sinh ^{-1} \frac{\mu_{3} z}{r}+\frac{\mu_{3}^{2}}{2\left(\mu_{3}^{2}-1\right)^{2}} \sinh ^{-1} \frac{\mu_{3} z}{r}, \\
\varphi_{4}(r, z)=\frac{1}{4\left(\mu_{3}^{2}-1\right)} \frac{z}{R}+\frac{1}{2\left(\mu_{3}^{2}-1\right)^{2}} \sinh ^{-1} \frac{z}{r}-\frac{1}{2\left(\mu_{3}^{2}-1\right)^{2}} \sinh ^{-1} \frac{\mu_{3} z}{r},
\end{gathered}
$$

where $R=\sqrt{r^{2}+z^{2}}$ and $R^{*}=\sqrt{r^{2}+z^{2}}+|z|$.

\subsection{Cases of Isotropic Mechanical and Thermal Properties}

Furthermore, the closed-form solutions for the special case when the properties of the medium is isotropic in mechanics and heat flows are acquired by taking the limit conditions of $\mu_{3}=1$ for Eqs. (19a) to (19c). Applying the L'Hospital's rule and careful calculations, the results are given as below:

$$
u_{r}(r, z)=\frac{Q}{8 \pi \lambda_{t}} \frac{(1+v) \alpha_{s}}{1-v} \frac{r}{R}
$$




$$
\begin{gathered}
u_{z}(r, z)=\frac{Q}{8 \pi \lambda_{t}} \frac{(1+v) \alpha_{s}}{1-v} \frac{z}{R}, \\
\vartheta(r, z)=\frac{Q}{4 \pi \lambda_{t}} \frac{1}{R},
\end{gathered}
$$

where $\lambda_{t}$ and $v$ represent the thermal conductivity and Poisson's ratio of the isotropic medium, respectively. It is noted from Eqs. (22a)-(22c) that the long-term horizontal displacement, vertical displacement and temperature increment of the stratum are not directly dependent on the shear modulus of the isotropic stratum. However, horizontal displacement and vertical displacement are dependent on the shear modulus of the crossanisotropic full space as shown in equations (16a)-(16b) or (19a)-(19b).

\section{Numerical Results}

To study the effect of anisotropy on displacements and temperature increment of the stratum due to a point heat source, numerical results have been obtained for different sets of thermoelastic constants appropriate for soils. The thermoelastic constants used are

\begin{tabular}{|c|c|c|c|c|c|c|c|}
\hline Case & $v_{r \theta}$ & $v_{r z}$ & $G_{r z} / E_{z}$ & $E_{r} / E_{z}$ & $\alpha_{s r} / \alpha_{s z}$ & $\lambda_{t r} / \lambda_{t z}$ & Reference \\
\hline Case 1 & 0.25 & 0.25 & 0.4 & 1.0 & $1.0^{*}$ & $1.0^{*}$ & $\begin{array}{l}\text { Booker \& Carter } \\
\text { (1986) }\end{array}$ \\
\hline Case 2 & 0.25 & 0.25 & 0.4 & 1.0 & $10.0^{*}$ & $10.0^{*}$ & $\begin{array}{l}\text { Booker \& Carter } \\
\text { (1986) }\end{array}$ \\
\hline Case 3 & 0 & 0.38 & 0.38 & 1.84 & $10.0^{*}$ & $10.0^{*}$ & $\begin{array}{l}\text { Lee \& Rowe } \\
(1989)\end{array}$ \\
\hline
\end{tabular}
summarized in Table 1.

Table 1. Material properties of cross-anisotropic soils ( ${ }^{*}$ assumed values)

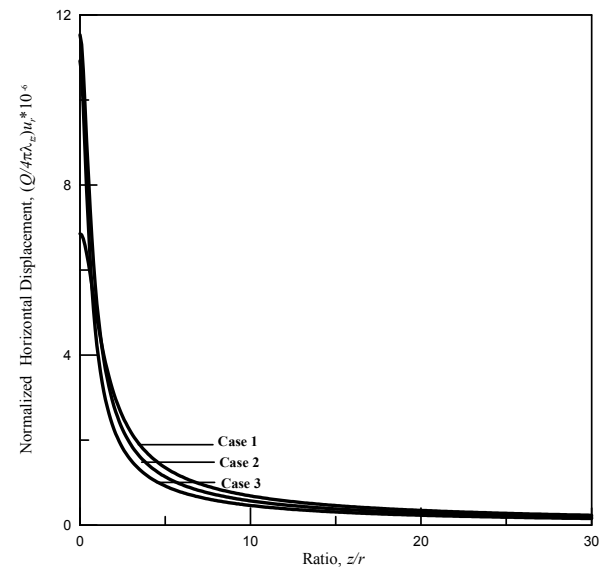

(a)

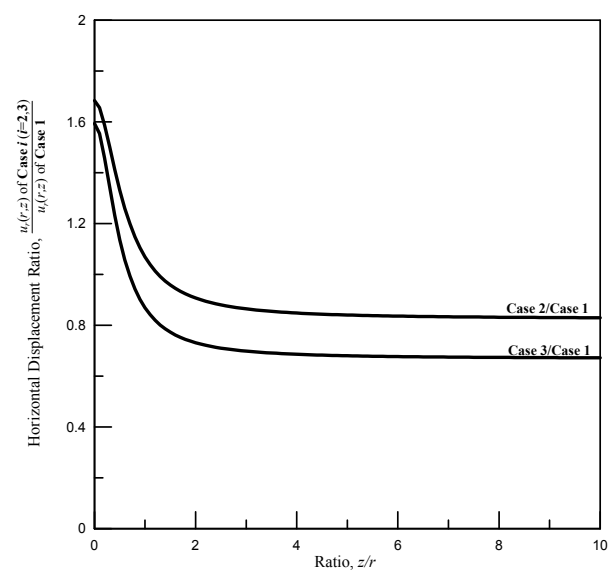

(b)

Fig. 2. Influence of anisotropy on horizontal displacement of the stratum 
An indication of the influence of anisotropy on the thermoelastic responses are given in Figures 2-4. In the figures, the thermoelastic responses have been normalized. It is observed from Figures 2-4 that the anisotropy of the soil has significant effect on thermally elastic responses in comparison with the results obtained for an isotropic soil of case 1 . For example, the vertical displacement of case 2 is reduced to about 60 percent of the corresponding value for the isotropic soil of case 1.

Figures 5-7 illustrate the horizontal displacement as effected by the anisotropy of the soil. As shown in Figure 5, the ratio $E_{r} / E_{z}$ ranges from 0.5 to 10.0, and the effect of $E_{r} / E_{z}$ on horizontal displacement of the stratum is secondary. Based on the available data, $v_{r \theta}=0.00$, $v_{r z}=0.38, G_{r z} / E_{z}=0.38$, and $\lambda_{t r} / \lambda_{t z}=1.0$ or 10.0, Figure 6 uses the ratio of $\alpha_{s r} / \alpha_{s z}$ to display the influence on horizontal displacement of the stratum. It is shown from Figure 7 that the degree of anisotropic thermal conductivity $\lambda_{t r} / \lambda_{t z}$ has the most significant effect on horizontal displacement of the stratum due to a point heat source.

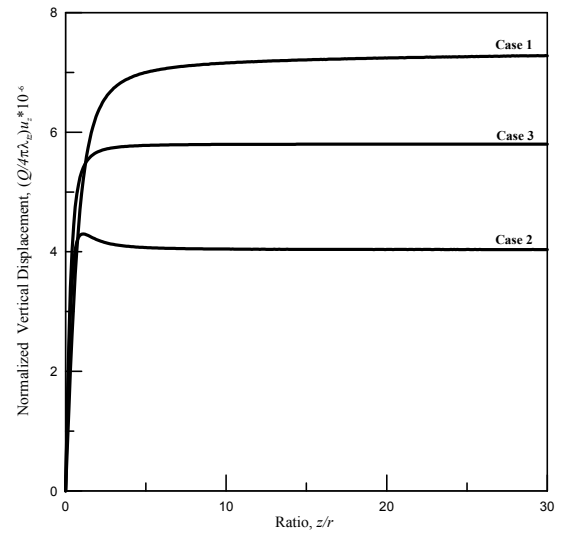

(a)

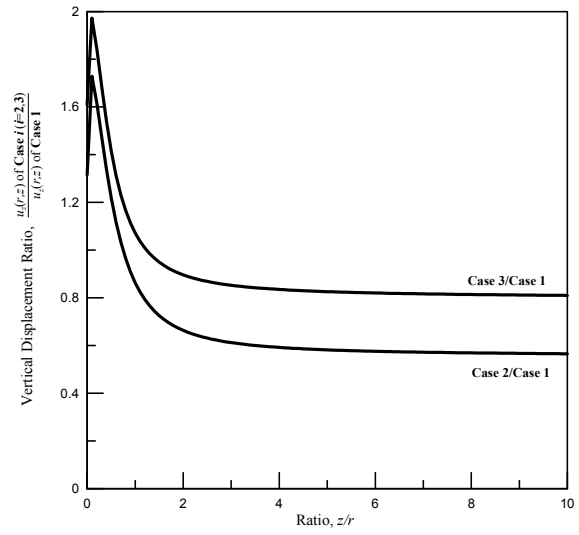

(b)

Fig. 3. Influence of anisotropy on vertical displacement of the stratum

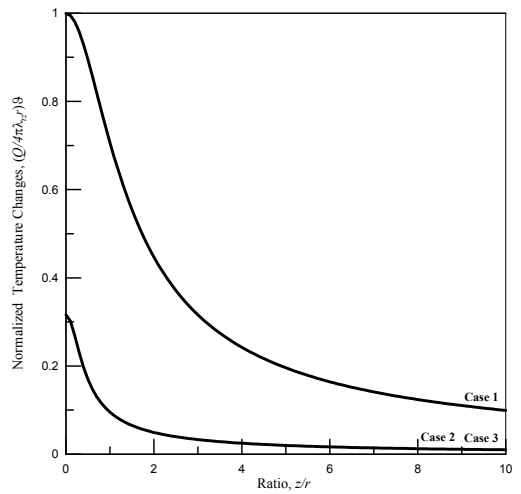

(a)

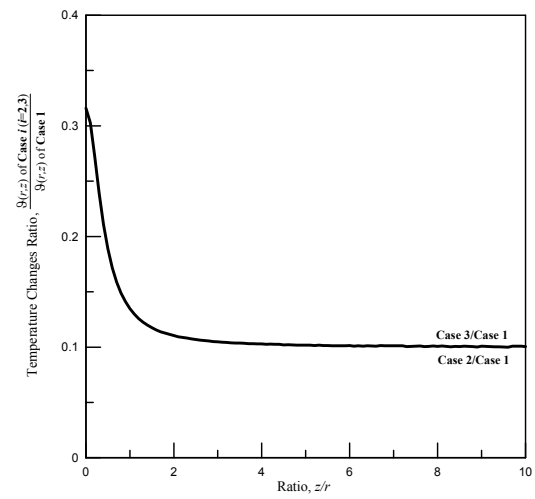

(b)

Fig. 4. Influence of anisotropy on the temperature increment of the stratum 


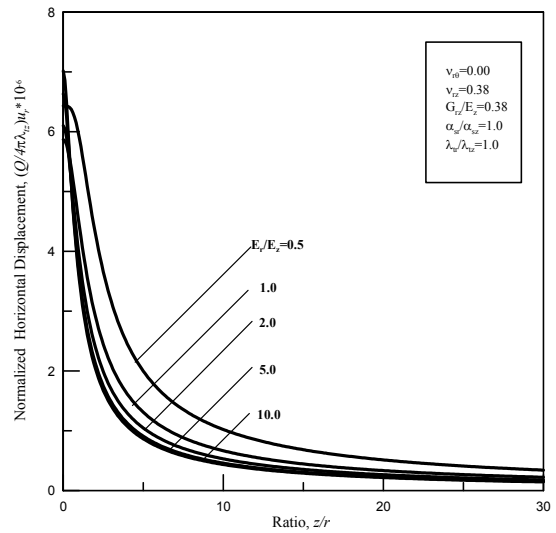

(a)

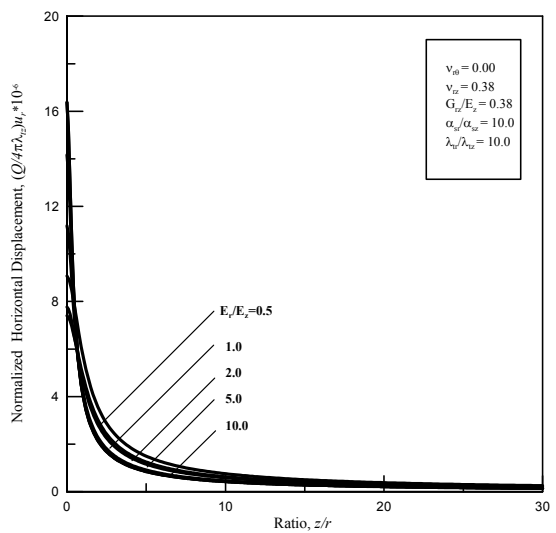

(b)

Fig. 5. Influence of the degree of anisotropy $E_{r} / E_{z}$ on horizontal displacement of the stratum

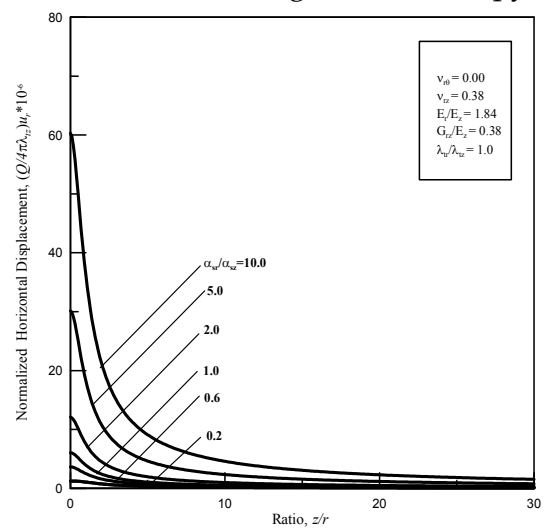

(a)

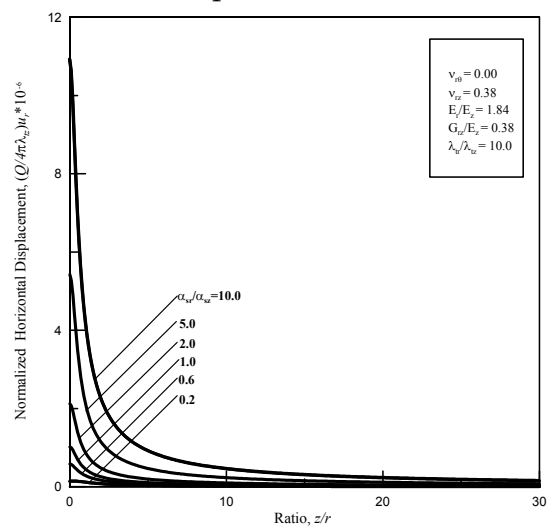

(b)

Fig. 6. Influence of the degree of anisotropy $\alpha_{s r} / \alpha_{s z}$ on horizontal displacement of the stratum

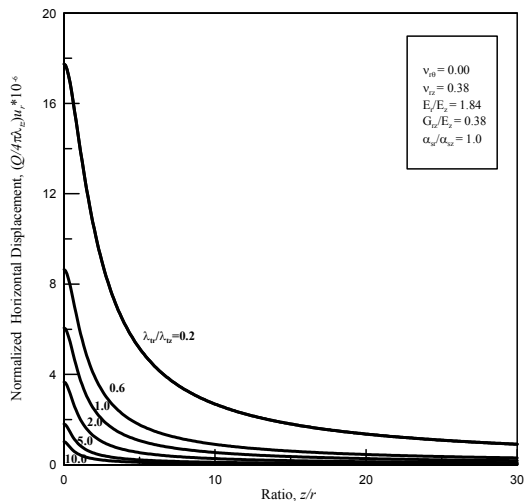

(a)

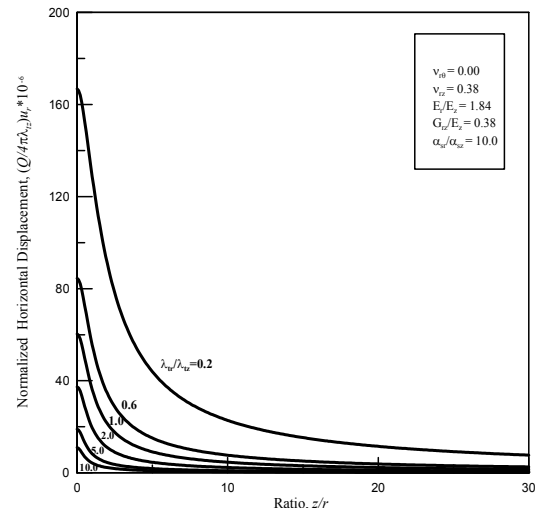

(b)

Fig. 7. Influence of the degree of anisotropy $\lambda_{t r} / \lambda_{t z}$ on horizontal displacement of the stratum 


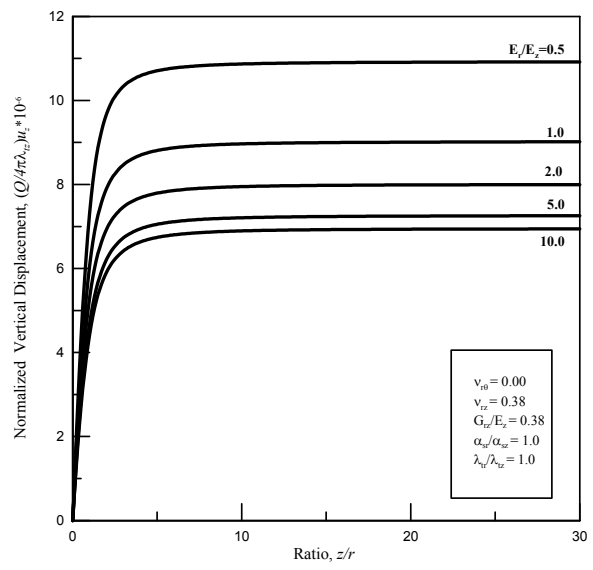

(a)

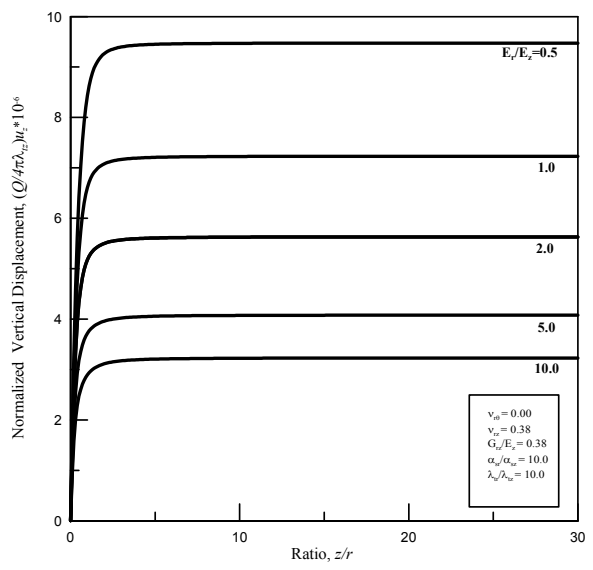

(b)

Fig. 8. Influence of the degree of anisotropy $E_{r} / E_{z}$ on vertical displacement of the stratum

Figures 8-10 show the vertical displacement as effected by the anisotropy of the soil. As shown in Figure 8, the effect of $E_{r} / E_{z}$ on vertical displacement of the stratum is significant for the ratio $E_{r} / E_{z}$ ranges from 0.5 to 10.0. Based on the available data, $v_{r \theta}=0.00, v_{r z}=0.38$, $G_{r z} / E_{z}=0.38, \lambda_{t r} / \lambda_{t z}=1.0$ or 10.0, Figure 9 uses the ratio of $\alpha_{s r} / \alpha_{s z}$ to display vertical displacement of the stratum. It is shown from Figure 10 that the degree of anisotropic thermal conductivity $\lambda_{t r} / \lambda_{t z}$ has the most significant effect on vertical displacement of the stratum due to a point heat source.

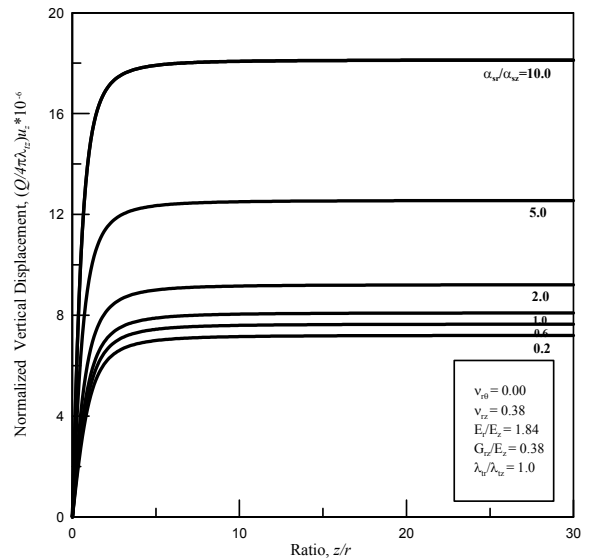

(a)

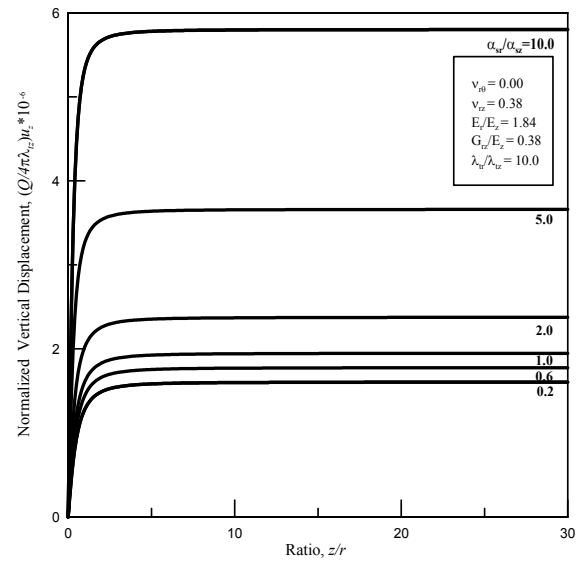

(b)

Fig. 9. Influence of the degree of anisotropy $\alpha_{s r} / \alpha_{s z}$ on vertical displacement of the stratum 


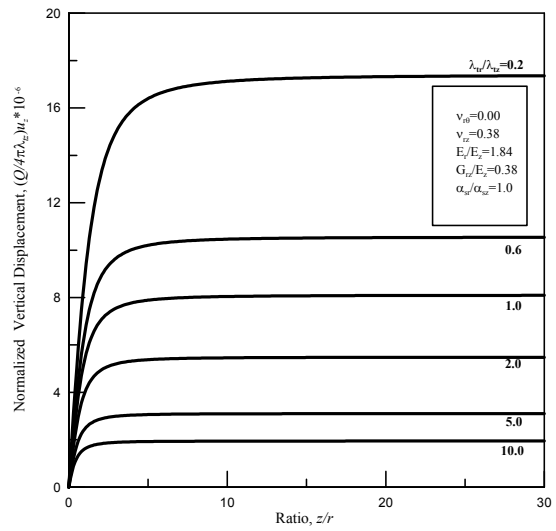

(a)

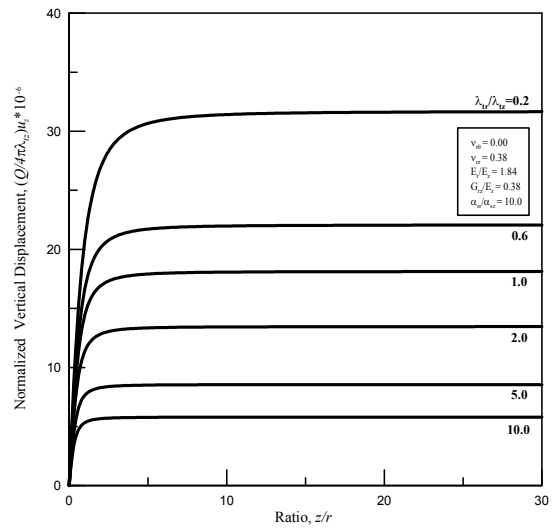

(b)

Fig. 10. Influence of the degree of anisotropy $\lambda_{t r} / \lambda_{t z}$ on vertical displacement of the stratum

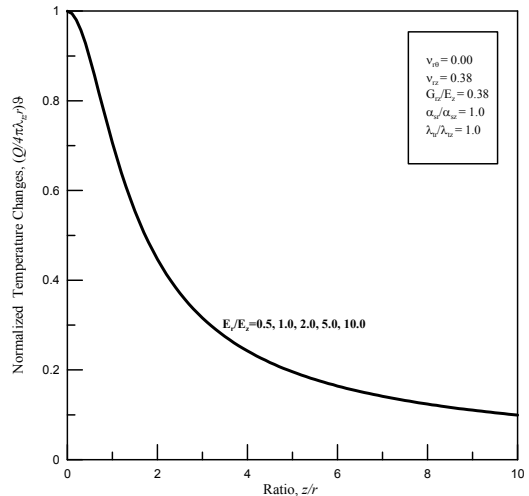

(a)

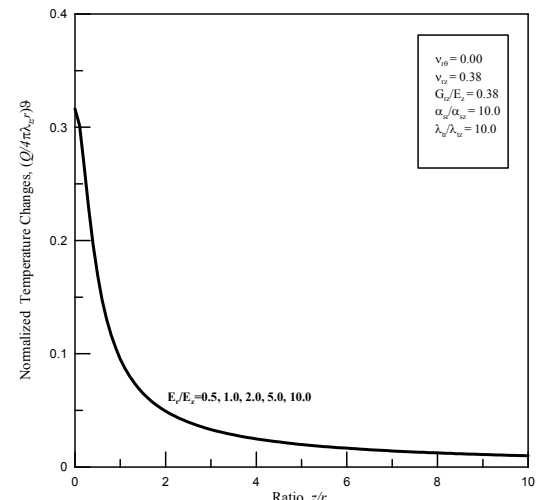

(b)

Fig. 11. Influence of the degree of anisotropy $E_{r} / E_{z}$ on temperature increment of the stratum

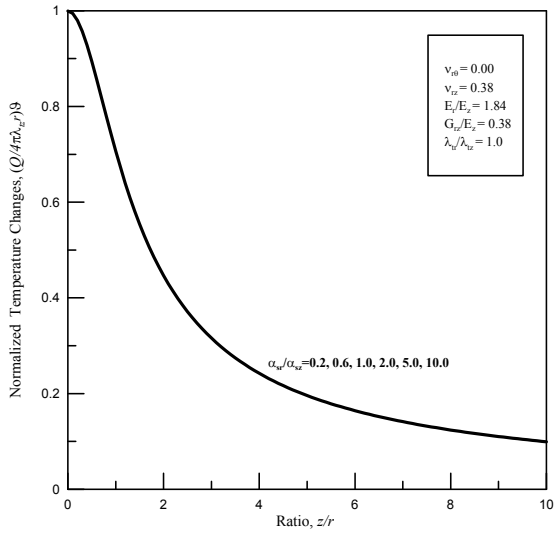

(a)

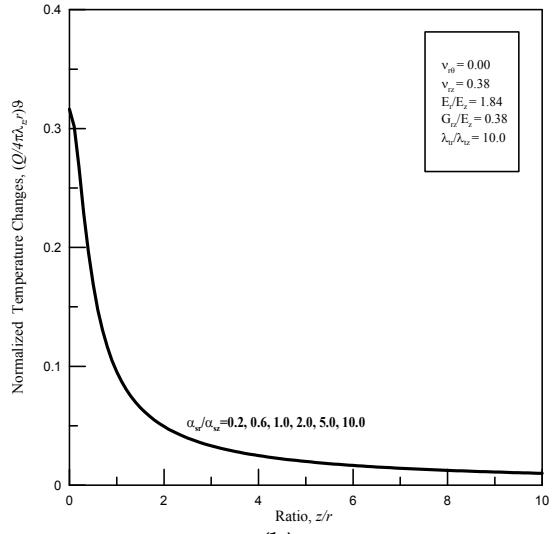

(b)

Fig. 12. Influence of the degree of anisotropy $\alpha_{s r} / \alpha_{s z}$ on temperature increment of the stratum 


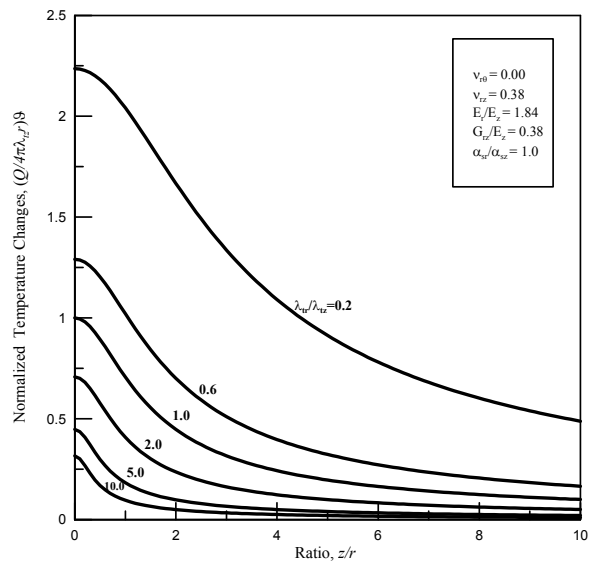

(a)

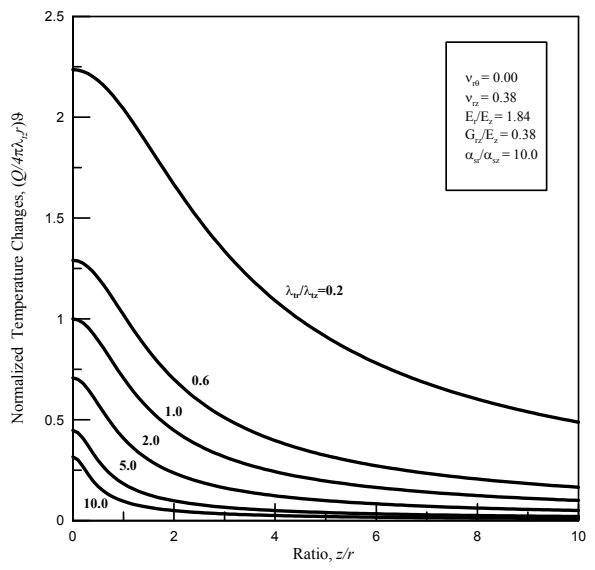

(b)

Fig. 13. Influence of the degree of anisotropy $\lambda_{t r} / \lambda_{t z}$ on temperature increment of the stratum

The temperature increments of the stratum were calculated from equation (16c) for values of various anisotropic ratio $E_{r} / E_{z}, \alpha_{s r} / \alpha_{s z}, \lambda_{t r} / \lambda_{t z}$ and the results are shown in Figures 11-13. Figures 11-12 display the anisotropic ratio $E_{r} / E_{z}$ and $\alpha_{s r} / \alpha_{s z}$ have no effect on the longterm temperature increment of the stratum due to a point heat source. However, Figure 13 illustrates that the ratio of anisotropic thermal conductivity $\lambda_{t r} / \lambda_{t z}$ has the most significant effect on temperature increment of the stratum. In all cases, the closer to the point heat source the larger is the temperature increment of the stratum.

\section{Conclusions}

This paper presents the mathematical modelling of a deep point heat source, such as the repositories of nuclear wastes. Using Hankel and Fourier integral transformations, analytic solutions of long-term thermo-mechanical responses of cross-anisotropic homogeneous soils or rocks due to a deep point heat source are obtained. The closed-form solutions of displacements and temperature change of the stratum are presented. The following conclusions were drawn based on the numerical results obtained for the available soil properties in Table 1:

1. It is noted from Eqs. (22a)-(22c) that the long-term horizontal displacement, vertical displacement and temperature increment of the stratum are not directly dependent on the shear modulus of the isotropic stratum. However, horizontal displacement and vertical displacement are dependent on the shear modulus of the cross-anisotropic full space as shown in equations (16a)-(16b) or (19a)-(19b).

2. The influence of anisotropy $E_{r} / E_{z}$ on horizontal displacement due to a point heat source is secondary while the effects of thermoelastic anisotropy $\alpha_{s r} / \alpha_{s z}$ or $\lambda_{t r} / \lambda_{t z}$ has primary effect on the horizontal displacement.

3. The influence of anisotropy $E_{r} / E_{z}, \alpha_{s r} / \alpha_{s z}$ and $\lambda_{t r} / \lambda_{t z}$ on vertical displacement subjected to a point heat source is of appreciable effect on the vertical displacement. 
Figures 11-12 show that the anisotropic ratio $E_{r} / E_{z}$ and $\alpha_{s r} / \alpha_{s z}$ have no effect on the long-term temperature increment of the stratum due to a point heat source. However, Figure 13 illustrates that the ratio of anisotropic thermal conductivity $\lambda_{t r} / \lambda_{t z}$ has the most significant effect on temperature increment of the stratum.

\section{Acknowledgements}

This work is supported by the National Science Council of Republic of China through grant NSC-98-2815-C-216-007-E, and also by the Chung Hua University under grant CHU-982815-C-216-007-E.

\section{References}

Amadei, B., Swolfs, H.S. \& Savage, W.Z. (1988). Gravity-induced stresses in stratified rock masses, Rock Mechanics and Rock Engineering, Vol. 21, No. 1, pp. 1-20.

Barden, L. (1963). Stresses and displacements in a cross-anisotropic soil, Geotechnique, Vol. 13, No. 2, pp. 198-210.

Biot, M.A. (1941). General theory of three-dimensional consolidation, Journal of Applied Physics, Vol. 12, No. 2, pp. 155-164.

Biot, M.A. (1955). Theory of elasticity and consolidation for a porous anisotropic solid, Journal of Applied Physics, Vol. 26, No. 2, pp. 182-185.

Booker, J.R. \& Savvidou, C. (1984). Consolidation around a spherical heat source, International Journal of Solids and Structures, Vol. 20, No. 11/12, pp. 1079-1090.

Booker, J.R. \& Savvidou, C. (1985). Consolidation around a point heat source, International Journal for Numerical and Analytical Methods in Geomechanics, Vol. 9, No. 2, pp. 173184.

Booker, J.R. \& Carter, J.P. (1986). Analysis of a point sink embedded in a porous elastic half space, International Journal for Numerical and Analytical Methods in Geomechanics, Vol. 10, No. 2, pp. 137-150.

Chao, C.K., Chen, F.M. \& Shen, M.H. (2006). Green's functions for a point heat source in circularly cylindrical layered media, Journal of Thermal Stresses, Vol. 29, No. 9, pp. 809 - 847.

Erdelyi, A.; Magnus, W., Oberhettinger, F. \& Tricomi, F.G. (1954). Tables of Integral Transforms, McGraw-Hill, New York.

Gibson, R.E. (1974). The analytical method in soil mechanics, Geotechnique, Vol. 24, No. 2, pp. 115-140.

Gradshteyn, I.S. \& Ryzhik, I.M. (1980). Table of Integrals, Series, and Products, Academic Press, 1160p.

Lee, S.L. \& Yang, J.H. (1998). Modeling of effective thermal conductivity for a nonhomogeneous anisotropic porous medium, International Journal of Heat and Mass Transfer, Vol. 41, No. 6-7, pp. 931-937.

Lee, K.M. \& Rowe, R.K. (1989). Deformations caused by surface loading and tunnelling: The role of elastic anisotropy, Geotechnique, Vol. 39, No. 1, pp. 125-140.

Lin, F.-T. \& Lu, J. C.-C. (2009). Analysis of transient ground surface displacements due to an instantaneous point heat source, Proceedings of the 20th IASTED International Conference on Modelling and Simulation, pp. 59-64, Banff, Alberta, Canada. 
Love, A.E.H. (1944). A Treatise on the Mathematical Theory of Elasticity, Dover Publications, New York, 643p.

Lu, J. C.-C. \& Lin, F.-T. (2006). The transient ground surface displacements due to a point sink/heat source in an elastic half-space, Geotechnical Special Publication No. 148, ASCE, pp. 210-218.

Lu, J. C.-C. \& Lin, F.-T. (2007). Thermo-consolidation due to a point heat source buried in a poroelastic half space, Proceedings of the $3^{\text {rd }}$ IASTED International Conference on Environmental Modelling and Simulation, pp. 48-53, Honolulu, Hawaii, U.S.A.

Rice, J.R. \& Cleary, M.P. (1976). Some basic stress diffusion solutions for fluid-saturated elastic porous media with compressible constituents, Reviews of Geophysics and Space Physics, Vol. 14, No. 2, pp. 227-241.

Savvidou, C. \& Booker, J.R. (1989). Consolidation around a heat source buried deep in a porous thermoelastic medium with anisotropic flow properties, International Journal for Numerical and Analytical Methods in Geomechanics, Vol. 13, No. 1, pp. 75-90.

Schiffman, R.L. (1971). A thermoelastic theory of consolidation, Environmental and Geophysical Heat Transfer, C.J. Cremers, et al., (eds.), ASME, Vol. 4, New York, pp. 78-84.

Sekowski, J. (1986). Stratified subsoil modelled by a cross-anisotropic elastic half-space, International Journal for Numerical and Analytical Methods in Geomechanics, Vol. 10, No. 4, pp. 407-414.

Sheorey, P.R. (1994). A theory for in situ stresses in isotropic and transversely isotropic rock, International Journal of Rock Mechanics and Mining Sciences and Geomechanics Abstracts, Vol. 31, No. 1, pp. 23-34.

Sneddon, I.N. (1951). Fourier Transforms, McGraw-Hill, New York, 542p.

\section{Notation of Symbols}

$\begin{array}{ll}a_{i}(i=1,2,3) & \text { Constants defined in equations (17a)-(17c) }\left({ }^{\circ} \mathrm{C}^{-1}\right) \\ A, C, F, L, N & \text { Material constants defined by Love }(\mathrm{Pa}) \\ b_{i}(i=1,2,3) & \text { Constants defined in equations }(17 \mathrm{~d})-(17 \mathrm{f})\left({ }^{\circ} \mathrm{C}-1\right) \\ E_{r}, E_{z} & \text { Young's modulus in horizontal/vertical direction }(\mathrm{Pa}) \\ f_{i}(i=r, \theta, z) & \text { Body forces of the stratum }\left(\mathrm{N} / \mathrm{m}^{3}\right) \\ G & \text { Shear modulus of the isotropic stratum }(\mathrm{Pa}) \\ G_{r z} & \text { Modulus of shear deformation in vertical plane }(\mathrm{Pa}) \\ \boldsymbol{h} & \text { Heat flux vector }\left(\mathrm{J} / \mathrm{sm}^{2}\right) \\ \boldsymbol{i}_{r}, \boldsymbol{i}_{z} & \text { Unit vector parallel to the radial/vertical direction (Dimensionless) } \\ J_{v}(x) & \text { First kind of the Bessel function of order } v \text { (Dimensionless) } \\ q_{h} & \text { Internal (or external) heat sources }\left(\mathrm{J} / \mathrm{sm} \mathrm{m}^{3}\right) \\ Q & \text { Strength of the point heat source }(\mathrm{J} / \mathrm{s}) \\ (r, \theta, z) & \text { Cylindrical coordinates system }(\mathrm{m}, \mathrm{radian}, \mathrm{m}) \\ R & \text { Parameter, } R=\sqrt{r^{2}+z^{2}}(\mathrm{~m}) \\ R_{i}(i=1,2,3) & \text { Parameter, } R_{i}=\sqrt{r^{2}+\mu_{i}^{2} z^{2}}(\mathrm{~m})\end{array}$




\begin{tabular}{|c|c|}
\hline$R^{*}$ & Parameter, $R^{*}=\sqrt{r^{2}+z^{2}}+|z|(m)$ \\
\hline$R_{i}^{*}(i=1,2,3)$ & Parameter, $R_{i}^{*}=R_{i}+\mu_{i}|z|(m)$ \\
\hline$u_{i}(i=r, \theta, z)$ & Displacement components of the stratum $(m)$ \\
\hline$U_{r}, U_{z}$ & Hankel transforms of $u_{r}$ and $u_{z}$, Eqs. (10a)-(10b) \\
\hline$\tilde{U}_{r}, \tilde{U}_{z}$ & Fourier transforms of $U_{r}$ and $U_{z}$, Eq. (13) \\
\hline$\alpha_{s}$ & Linear thermal expansion coefficient of the isotropic stratum $\left({ }^{\circ} \mathrm{C}^{-1}\right)$ \\
\hline$\alpha_{s r}, \alpha_{s z}$ & $\begin{array}{l}\text { Linear thermal expansion coefficient of the cross-anisotropic stratum in } \\
\text { horizontal/vertical direction }\left({ }^{\circ} \mathrm{C}^{-1}\right)\end{array}$ \\
\hline$\beta_{r}, \beta_{z}$ & Thermal expansion factors of the cross-anisotropic stratum $\left(\mathrm{Pa} /{ }^{\circ} \mathrm{C}\right)$ \\
\hline$\beta_{r}^{*}, \beta_{z}^{*}$ & Thermal expansion factors of the isotropic stratum $\left(\mathrm{Pa} /{ }^{\circ} \mathrm{C}\right)$ \\
\hline$\delta(x)$ & Dirac delta function $\left(m^{-1}\right)$ \\
\hline$\eta$ & Parameter, $\eta=(1-v) /(1-2 v)$ (Dimensionless) \\
\hline$\vartheta$ & Temperature change of the stratum $\left({ }^{\circ} \mathrm{C}\right)$ \\
\hline$\Theta$ & Hankel transform of $\vartheta$, Eq. (10c) \\
\hline$\tilde{\Theta}$ & Fourier transform of $\Theta$, Eq. (13) \\
\hline$\lambda$ & Lame constant of the isotropic stratum $(\mathrm{Pa})$ \\
\hline$\lambda_{t}$ & Thermal conductivity of the isotropic thermoelastic medium $\left(\mathrm{J} / \mathrm{sm}^{\circ} \mathrm{C}\right)$ \\
\hline$\lambda_{t r}, \lambda_{t z}$ & $\begin{array}{l}\text { Thermal conductivity of the cross-anisotropic thermoelastic medium in } \\
\text { the horizontal/vertical direction }\left(\mathrm{J} / \mathrm{sm}^{\circ} \mathrm{C}\right)\end{array}$ \\
\hline$\mu_{1}, \mu_{2}$ & Characteristic roots of characteristic equation (18) (Dimensionless) \\
\hline$\mu_{3}$ & Characteristic root, $\mu_{3}=\sqrt{\lambda_{t r} / \lambda_{t z}}$ (Dimensionless) \\
\hline$v$ & Poisson's ratio of the isotropic stratum (Dimensionless) \\
\hline$v_{r z}$ & $\begin{array}{l}\text { Poisson's ratio for strain in the vertical direction due to a horizontal } \\
\text { direct stress (Dimensionless) }\end{array}$ \\
\hline$v_{r \theta}$ & $\begin{array}{l}\text { Poisson's ratio for strain in the horizontal direction due to a horizontal } \\
\text { direct stress (Dimensionless) }\end{array}$ \\
\hline$v_{z r}$ & $\begin{array}{l}\text { Poisson's ratio for strain in the horizontal direction due to a vertical } \\
\text { direct stress (Dimensionless) }\end{array}$ \\
\hline$\xi$ & Hankel transform parameter $\left(m^{-1}\right)$ \\
\hline$\sigma_{i j}(i, j=r, \theta, z)$ & Thermal stress components of the stratum $(\mathrm{Pa})$ \\
\hline$\varphi_{i}(i=1,2,3,4)$ & Functions defined in Eqs. (21a)-(21d) (Dimensionless) \\
\hline$\omega$ & Fourier transform parameter $\left(m^{-1}\right)$ \\
\hline
\end{tabular}




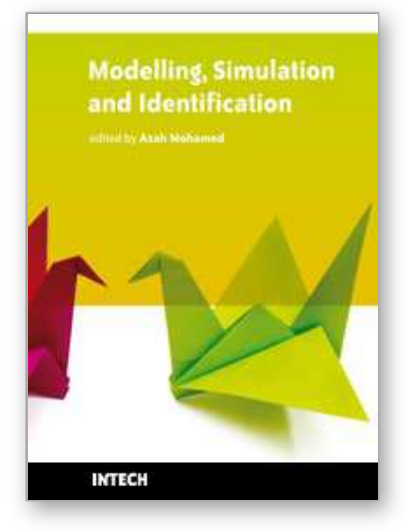

\author{
Modelling, Simulation and Identification \\ Edited by Azah Mohamed
}

ISBN 978-953-307-136-7

Hard cover, 354 pages

Publisher Sciyo

Published online 18, August, 2010

Published in print edition August, 2010

Modeling, simulation and identification has been actively researched in solving practical engineering problems. This book presents the wide applications of modeling, simulation and identification in the fields of electrical engineering, mechanical engineering, civil engineering, computer science and information technology. The book consists of 17 chapters arranged in an order reflecting multidimensionality of applications related to power system, wireless communication, image and video processing, control systems, robotics, soil mechanics, road engineering, mechanical structures and workforce capacity planning. New techniques in signal processing, adaptive control, non-linear system identification, multi-agent simulation, eigenvalue analysis, risk assessment, modeling of dynamic systems, finite difference time domain modeling and visual feedback are also presented. We hope that readers will find the book useful and inspiring by examining the recent developments in the applications of modeling, simulation and identification.

\title{
How to reference
}

In order to correctly reference this scholarly work, feel free to copy and paste the following:

Feng-Tsai Lin and John C.-C. Lu (2010). Closed-Form Solutions of the Cross-Anisotropic Stratum Due to a Point Heat Source, Modelling, Simulation and Identification, Azah Mohamed (Ed.), ISBN: 978-953-307-136-7, InTech, Available from: http://www.intechopen.com/books/modelling--simulation-and-identification/closed-formsolutions-of-the-cross-anisotropic-stratum-due-to-a-point-heat-source

\section{INTECH}

open science | open minds

\author{
InTech Europe \\ University Campus STeP Ri \\ Slavka Krautzeka 83/A \\ 51000 Rijeka, Croatia \\ Phone: +385 (51) 770447 \\ Fax: +385 (51) 686166 \\ www.intechopen.com
}

\author{
InTech China \\ Unit 405, Office Block, Hotel Equatorial Shanghai \\ No.65, Yan An Road (West), Shanghai, 200040, China \\ 中国上海市延安西路65号上海国际贵都大饭店办公楼405单元 \\ Phone: +86-21-62489820 \\ Fax: +86-21-62489821
}


(C) 2010 The Author(s). Licensee IntechOpen. This chapter is distributed under the terms of the Creative Commons Attribution-NonCommercialShareAlike-3.0 License, which permits use, distribution and reproduction for non-commercial purposes, provided the original is properly cited and derivative works building on this content are distributed under the same license. 